\title{
PROCESO DE REFLEXIVIDAD EN UN CONTEXTO DE PRIVACIÓN DE LIBERTAD DE ADOLESCENTES MUJERES
}

PROCESSO DE REFLEXIVIDADE EM UM CONTEXTO DE PRIVAÇÃO DE LIBERDADE DE MULHERES ADOLESCENTES REFLEXIVITY PROCESS IN THE INCARCERATION OF TEENAGE GIRLS

\author{
Laura López Gallego \\ Universidad Autónoma de Barcelona, Barcelona, España y Universidad de la República, Montevideo, \\ Uruguay
}

\begin{abstract}
RESUMEN
A través del presente artículo, reflexionamos acerca de algunos acontecimientos, hallazgos e itinerarios del trabajo de campo de tesis doctoral que realizamos en el Sistema Penal Juvenil Uruguayo (SPJU), más específicamente en el escenario institucional de las adolescentes mujeres que cumplen una medida judicial en régimen de encierro. Discutimos acerca de las singularidades teórico-metodológicas de un proyecto de investigación, donde la relación de la investigadora-adolescentes es centro del debate. Analizamos un caso, "Lucrecia", para ilustrar la discusión en torno a la escritura y la reflexividad, así como la perspectiva de género en el marco del SPJU. Finalmente, nos preguntamos por el proceso de reflexividad en la investigación cualitativa y su relevancia vinculada a las posibilidades de análisis del vínculo investigador/a-participantes en investigaciones realizadas en contexto de encierro.
\end{abstract}

Palabras claves: sistema penal juvenil; investigación cualitativa; prácticas de escritura; género; reflexividad.

\section{RESUMO}

Através deste artigo, refletimos sobre alguns eventos, resultados e itinerários do trabalho de campo para tese de doutorado realizado no Sistema Penal Juvenil Uruguaio (SPJU), mais especificamente no contexto institucional de mulheres adolescentes que cumprem uma decisão judicial em regime de confinamento. Discutimos as peculiaridades teóricas e metodológicas de um projeto de pesquisa, em que a relação pesquisadora-adolescentes de pesquisa é o centro do debate. Analisamos um caso, "Lucrecia" para ilustrar a discussão sobre a escrita e a reflexividade, bem como a perspectiva de gênero sob o SPJU. Finalmente, nos questionamos sobre o processo de reflexividade na pesquisa qualitativa e sua relevância relacionada às possibilidades de análise do vínculo pesquisador/a - participantes na pesquisa realizada em contexto de confinamento.

Palavras-chave: sistema penal juvenil; pesquisa qualitativa; práticas de escrita; gênero; reflexividade.

\begin{abstract}
Through this article, we reflect on certain events, results and itineraries of the fieldwork of Phd thesis produced in the Juvenile Justice System of Uruguay, more specifically in the institutional setting of teenage women serving a confinement sentence. We discussed the theoretical and methodological peculiarities of a research project, where the relationship between the researcher-teenager is focused. We analyze a case, "Lucrecia" to illustrate the discussion of writing and reflexivity, as well as the gender perspective under the SPJU. Finally, we asked for the process of reflexivity in qualitative research and its relevance related to the potential for the analysis of the linkage between researcher/participants in research conducted in context of confinement.
\end{abstract}

Keywords: juvenile justice system; qualitative research; writing practices; gender; reflexivity. 


\section{Acerca de la escritura y la reflexividad en un proceso de investigación cualitativa}

La propuesta de este artículo es compartir algunas reflexiones en torno a nuestro trabajo de campo que se desarrolló con las adolescentes mujeres residentes en una institución de encierro, única en el marco del Sistema Penal Juvenil Uruguayo (SPJU). El proyecto de investigación, en términos generales, pretende comprender el funcionamiento de las medidas judiciales en régimen de encierro aplicadas a adolescentes mujeres en el SPJU, en términos de los objetivos que se proponen, los medios que disponen para conseguirlos, los agentes y tecnologías con las que cuentan y los efectos que generan.

El enfoque metodológico es cualitativo (Denzin \& Lincoln, 2003; Olabuénaga, 1996/2009). Privilegiamos los significados intersubjetivos que se producen en la interacción humana en un determinado contexto socio-histórico. Esta impronta históricosimbólica, dará al lenguaje un papel clave, en tanto implica la construcción y circulación de significados. Lo que se ha llamado giro lingüistico, remarca la naturaleza social del lenguaje, emergiendo en la acción e interacción humana como una actividad de carácter performativo. El lenguaje, de esta forma ya no representa y/o refleja, sino que actúa y constituye los fenómenos sociales. De esta forma, las explicaciones que los actores sociales formulan sobre su existencia cotidiana conforman un material privilegiado en la búsqueda de comprensión de las relaciones sociales (Iñiguez, 2003).

En este contexto, donde las prácticas lingüísticas ocupan un lugar clave, nos preguntamos, en primer término, por las posibilidades, características y desafíos que implica la escritura de un trabajo de investigación realizado en una institución de encierro para adolescentes mujeres. Hacemos acuerdo con Billig (1994) cuando nos dice que las formas de escritura construyen a la Psicología Social.

En su artículo "Repopulating the Depopulated Pages of Social Psychology" publicado en el año 1994, problematiza las formas de escritura de artículos en revistas de psicología social experimental. Analiza dos números de la European Journal of Social Psychology (1991), concluyendo que la retórica predominante en estos textos utiliza estrategias para despoblar a las personas de los informes de investigación. A lo que agrega que también en otras disciplinas sociales, o en las vertientes más críticas de la Psicología Social podemos encontrar el mismo tipo de retórica, poblada de personas convertidas en abstracciones descorporizadas, intercambiables unas por otras.

El trabajo de campo es claramente construido con diversas personas: conversaciones, entrevistas, observaciones, un proceso dialógico donde participan múltiples sujetos. Ahora bien, la pregunta que nos surge es ¿cómo no convertir todo aquello en generalizaciones intercambiables entre sujetos homogéneos?

En segundo término, los postulados recientes de la IC nos hablan de un clima de horizontalidad entre investigador/a-investigado/a, tendiente a la simetría, el diálogo y el respeto mutuo. En este marco, la reflexividad del investigador es una condición de producción de conocimiento (Sisto, 2008). La investigación cualitativa es pensada como un proceso co-construido, donde la subjetividad del investigador se pone en juego en forma activa y reflexiva en dicho proceso. La reflexividad es entendida como "el ejercicio de una conciencia crítica respecto de la propia acción del investigador como sujeto realizador de la investigación. La reflexividad hace del investigador y de su actividad objeto de su propio análisis" (Sisto, 2008, p.132). ¿Cómo efectivizar estos postulados de las metodologías cualitativas en una investigación realizada en una institución de encierro?

Las dificultades son muchas, el contexto de encierro marca asimetrías: es la investigadora la que selecciona el contexto de trabajo en función de sus propósitos de investigación, negocia los permisos y accede a algo de la vida institucional, en un contexto donde los derechos de las personas están sumamente vulnerados. La asimetría es grande, la responsabilidad para con la reflexividad también lo es.

García (2006) analiza el concepto de reflexividad en la obra de Bourdieu. Reflexiona acerca de cómo se va emplazando la práctica de la reflexividad en el terreno de la ética, "entendiendo por tal, no un código o una deontología profesional propia de los investigadores sociales, sino la tarea empeñada por éstos en constituirse como sujetos de conocimiento y, al mismo tiempo, involucrase en una acción política y moral" (p.88).

En este sentido, la reflexividad debe abarcar el conocimiento que se construye con ellas pero que nosotros lo escribimos sin ellas. Convertirlas en categorías/tipos homogéneos de delincuentes adolescentes mujeres sería un desastre como producto narrativo y sobre todo como acción éticopolítica. Algunas autoras (Patai, 1991; Pillow, 2003) advierten acerca del riesgo de convertir el proceso de reflexividad en un cuento confesional por parte del 
investigador/a, que intente situar al investigador/a próximo y/o familiar con los/as participantes de la investigación.

Desde una posición ético-epistemológica, Haraway (1995) apela a los conocimientos situados, en tanto que parciales, localizables y críticos, posibilitan una objetividad encarnada que se posiciona para construir versiones posibles de la realidad estudiada.

Así, de manera no tan perversa, la objetividad dejará de referirse a la falsa visión que promete la trascendencia de todos los límites y responsabilidades, para dedicarse a una encarnación particular y específica. La moraleja es sencilla: solamente la perspectiva parcial promete una visión objetiva. Se trata de una visión objetiva que pone en marcha, en vez de cerrar, el problema de la responsabilidad para la generatividad de todas las prácticas visuales. (Haraway, 1995, p. 326)

El desafío es poder integrar el problema de la responsabilidad no sólo a las prácticas visuales sino que también a las prácticas de escritura. El peligro creemos está en construir un conocimiento que dista de los significados que ellas mismas le otorgan a sus sentimientos, pensamientos, prácticas cotidianas, desconociéndolas para convertirlas en sujetos estigmatizados (Weisenfeld, 2000). Como no caer en categorías explicativas que reifican la pobreza, el delito y el abuso en términos de relación lineal causaefecto, es una de nuestras apuestas.

Como itinerario teórico-metodológico, pretendemos otros caminos de lo particular a lo general, que habiliten transferencias diversas de conocimiento. En este artículo, analizamos un caso, "Lucrecia", para ilustrar cómo lo particular puede servir para analizar prácticas de reflexividad y escritura. Tenemos presente lo que Spivak (1988) afirma acerca de que un posicionamiento más "transparente" no lo torna en una cuestión sin problemas, sino que todo lo contrario, desafía al investigador/a a trabajar con su propia incomodidad y angustia, lo que Devereux (1977/1989) llama contratransferencia.

En este marco, dos propósitos tiene este artículo. En primer lugar, realizar un ejercicio de reflexividad que muestre algunas de las incertidumbres y los hallazgos que este proyecto de tesis conlleva y desafíe el contexto confesional al que está la relacionada la reflexividad en la investigación cualitativa en la actualidad. En segundo lugar, pensar en cómo escribimos acerca de ellas y nosotros, de modo de visibilizar algunos aspectos de la temática que abordamos: la investigación cualitativa en una institución de encierro de adolescentes mujeres.

\section{Consideraciones metodológicas: acerca de la viabilidad de investigar en instituciones de encierro de adolescentes mujeres en el SPJU}

La pregunta por la viabilidad en este tipo de investigación es clave. ¿Cómo acceder a estos espacios, que se caracterizan por el encierro y el aislamiento de sus habitantes? Luego de más de un año de negociaciones, de puertas cerradas y porteros varios, podemos comenzar a pensar en nuestro mapa de ruta, gracias a que el nuevo director que asume, proveniente de la academia, considera valioso que se realicen investigaciones. El trabajo de campo duró un año aproximadamente entre abril de 2010 y julio de 2011. El trabajo propiamente con las adolescentes mujeres se realizó en cinco meses de permanencia en la institución, con una frecuencia bisemanal.

El concepto de portero (Hammersly \& Atkinson, 1983/1994), tan utilizado en los métodos etnográficos, parece clave para calibrar la viabilidad de investigar en instituciones de encierro. Parte del trabajo de investigación implica el conocimiento de quién puede facilitar y/o bloquear el acceso al lugar. Asimismo, reflexionar acerca de los obstáculos que traban el acceso, así como de las formas de sortearlos, nos da pistas del funcionamiento institucional. En este caso caracterizado por la rotación y discrecionalidad de las personas que ocupan cargos de dirección.

En este contexto, surgen cuestiones a pensar: cuán próximos al portero podemos quedarnos? Cuánto de eso que habilitó en un inicio y abrió una puerta, puede cerrar puertas a futuro? Obtener un permiso de una autoridad es sólo el primer paso de la entrada al campo. Taylor y Bogdan (1984/1992) advierten sobre la necesidad de tomar distancia de los porteros una vez que comienza el proceso de investigación, de modo de no quedar unívocamente relacionados a ellos por lo que pueden generar en el resto de las personas. En nuestro caso, el portero es nada menos que el nuevo director, por lo cual la tensión se genera acerca de cuál es nuestro lugar ¿investigar es igual a evaluar la institución en cuanto a su funcionamiento?

Por otra parte, cabe resaltar que durante el tiempo que duró el trabajo de campo no tuvimos ninguna injerencia y/o control por su parte, lo que habilitó a no quedar fijados en ese vínculo e ir tejiendo diversas relaciones de campo de forma gradual, hasta lograr un nivel de confianza con las diversas personas que permitió, en muchos momentos, ser una participante más de la institución, con restricciones de tiempos y tareas. 
En esta institución, viven alrededor de 20 adolescentes en promedio. Dado los permisos obtenidos en términos de tiempo para la investigación, no más de 6 meses dentro de la institución, y la permanencia de las adolescentes en la misma, fue difícil encontrarnos con todas ellas. La muestra, algo tan importante para el mundo de la investigación, comienza a ser tema. Definimos algunos criterios antes y otros surgen en el trabajo con ellas: la edad que de acuerdo al Código de la Niñez y Adolescencia (Lei $\mathrm{n}^{\mathrm{o}}$ $17.823,2004)$ se divide en dos grupos de 13 a 15 años y de 16 a 17 años de edad; la procedencia geográfica (Capital del País y Resto del País); la condición de primera vez o reincidente en el SPJU; y, por último, la condición de tener hijos o no. En el marco del trabajo de campo, sumamos el criterio de estar embarazada o no, en función de las diferencias que genera, por ejemplo, el embarazo determina que no se consuman psicofármacos.

Para este artículo, hemos elegido a una adolescente. Siguiendo los consejos de Billig (1994), nos animamos a ensayar otras formas de escritura que singularizan, en definitiva encarnan, la temática. ¿Por qué una adolescente? Por un tema de espacio y tiempo. ¿Por qué ella? Por el impacto afectivo que nos generó, pero también por lo que nos hizo pensar teórica y metodológicamente. Sabemos que hemos dejado mucho de lado, las otras ochos adolescentes y las autoridades, los técnicos y los educadores del lugar. El reto es poder a través de ella, Lucrecia, pensar la reflexividad y la escritura, en el marco de los desafíos que una investigación de estas características conlleva.

\section{Narrando la reflexividad: Lucrecia}

El lugar que me asignan para realizar entrevistas es el salón contiguo al taller de costura. Tiene timbre por dentro, lo que significa que la puerta se cierra por fuera. Yo también estoy encerrada durante las entrevistas. En un primer momento, la situación me impacta. Luego me acostumbro y debo de tocar timbre y esperar para salir, o para que alguna de las adolescentes salga.

El salón que me asignan es pequeño. $\mathrm{Su}$ mobiliario está compuesto por dos sillas y una mesa, en las paredes no hay nada a excepción de una cartelera de espuma con alfileres. Intento hacerme la idea de que si esos alfileres están ahí es porque no debe ser peligroso -aunque esto no logra tranquilizarme.

La coordinadora me pregunta si me voy a quedar con mis objetos personales en la entrevista (un bolso que contiene cuaderno, lapicera, grabador, billetera y teléfono móvil). Le respondo que sí y me dice que es a mi riesgo. Vuelvo a reparar en los alfileres. Comienzo la entrevista tensa.

La construcción de las adolescentes como peligrosas está presente, a nivel institucional, en el tipo de preguntas y resguardos que tienen para conmigo y en el lugar que me asignan. En mí, a través del miedo que esta pregunta y el lugar -cerrado por fuera- me suscita. Desde una perspectiva genealógica, el complejo tutelar que opera en el siglo $\mathrm{XX}$, como lo denomina Donzelot (1977/2008), focaliza en la construcción de esos niños en peligro de convertirse en peligrosos. El objetivo privilegiado, la infancia patologizada, comparte un doble registro: la infancia en peligro porque no tuvo las condiciones óptimas de crianza y educación y la infancia peligrosa, aquella vinculada a la delincuencia.

\section{Lucrecia: en su primera semana}

Es mi primera entrevista con una adolescente, por lo que estoy bastante nerviosa y con miedos varios. El lugar no ayuda mucho a calmarme, quien sí me ayuda es Lucrecia. Ella llega con muchas ganas de hablar y comienza a contarme su vida sin más introducción que mi garantía de confidencialidad y la explicación de los motivos de esta entrevista: mi proyecto de investigación. Me gusta que me hagas preguntas, me dice, lo cual me facilita el trabajo al permitirme una conexión fácil con ella.

Sus primeras palabras vehiculizan una necesidad, hablar con la asistente social para saber cómo está su hijo. Desde el comienzo, mi posición en relación a ella intenta que la situación que vive no sea tan angustiante. Se siente preocupada dado que no ha tenido noticias de él. El niño tiene 11 meses y me cuenta que tomaba teta antes de que ella estuviera aquí. Vivían juntos, con su madre, en una casa de propiedad familiar.

- No por eso quiero hablar con la asistente social, con mi familia se que no voy a contar y con mi madre capaz que sí, porque mamá es un pan de dios ¿viste?

- El tema es que querés saber de tu hijo.

- Primero tengo que saber de mi hijo, es lo primero que quiero saber porque me debe de extrañar y todo. Sabes que yo tengo miedo de que yo salga de acá a la calle y no me conozca, me quiero matar! Porque es un bebé, un bebé se adapta enseguida.

\footnotetext{
- No, no pero no es tan rápido Lucrecia, vivió siempre contigo?

$-Y s i$
} 
Hace una semana que llegó a la institución y viene de una ciudad ubicada en el norte del país, es decir su casa, su familia y su hijo se encuentran lejos de aquí. Me hace pensar en la maternidad en el encierro. Cuál de las violencias es la peor para el niño: estar encerrado junto a su madre o verse sometido a un destete y separación abrupta. La realidad actual de encierro en adolescentes mujeres no hace la decisión fácil, no está preparada la institución para que las adolescentes vivan con sus hijos, solamente se han realizado excepciones cuando el niño nace mientras su madre está internada. Como tantas veces en el sistema penal adolescente no hay protocolos, lo cual determina que es la discrecionalidad de ciertas personas que dirigen las instituciones, las que terminan decidiendo qué hacer, en este caso con los hijos de las adolescentes.

Igareda (2009) concluye en que las mujeres presas que no pueden estar con sus hijos se sienten incompletas como mujeres, fallidas en su función de madres. En la actualidad, aún priman modelos donde la mujer se construye identitariamente en relación a ser madre. "Se es mujer en la medida en que se llega a ser madre y no se puede hablar de una mujer plena hasta que no ha cumplido con la función social por excelencia: ser madre" (p. 162).

Comienza a hablar de lo que la trajo aquí, me dice que se drogaba desde antes de cumplir los 15 , ahora tiene 17 años sólo llegaba a casa a comer y dormir, estaba todo el día en la calle. Los problemas con su madre llegaron a situaciones de violencia física, confiesa que le pegaba para conseguir lo que quería; dinero para seguir consumiendo. Cuando me está contando esto, observo su cuerpo, es realmente grande, alta, gorda, muy corpulenta, imagino lo difícil de un enfrentamiento físico con ella. En la última pelea, su madre terminó internada, aparentemente se quiso suicidar y la encontraron muy grave. El relato está cargado de emociones, las lágrimas asoman en los ojos de Lucrecia; se pregunta si su madre la podrá perdonar.

El delito: lesiones personales. Está encerrada por golpear a su madre, me sorprende, no lo esperaba, mis propios prejuicios se juegan en cada diálogo. Soy hija y soy madre ¿cómo hacer para que esto no me obture las posibilidad de comprender?

Pillow (2003) reflexiona acerca del uso de la reflexividad en términos de la subjetividad del investigador/a. Quién soy y cómo estos datos me afectan son parte de esa forma de utilizar la reflexividad, que se asemeja a una confesión narcisística. Propone una alternativa crítica a ese uso de la reflexividad que puede situar la dificultad y la incomodidad en el terreno de lo no familiar para el/la investigador/a. En términos de Devereux (1989), la contratransferencia no debe intentar posicionarme en términos de similitudes con Lucrecia, sino que, por el contrario, debe de poder mostrar nuestras diversidades y el impacto que ello me ocasiona. No debo apelar a concepciones unívocas acerca de la maternidad, porque sin duda eso dificulta mis posibilidades de comprender a Lucrecia

not to situate reflexivity as a confessional act, a cure for what ails us, or a practice that renders familiarity, but rather to situate practices of reflexivity as critical to exposing the difficult and often uncomfortable task of leaving what is unfamiliar, unfamiliar. (Pillow, 2003, p.177)

La violencia física y el abuso sexual surgen en su relato. Su papá siempre le pegó, "terribles palizas" sin mucho motivo. Vivió con sus padres hasta la separación de ambos, cuando ella tenía 14 años. Me cuenta que es hija adoptiva y que cuando tenía 11 años su abuelo materno trató de abusar sexualmente de ella y se escapó.

-Si, y agarre y vino y empezó a tocarme de nuevo y me pare yo viste. Y el vino así y me seguía tocando arriba viste, cuando empezó a tocar abajo lo empuje y sali.

-Te fuiste...

-Sabes cuál es el tema, que yo asustada no le quería decir a nadie, ¿viste?

Salí corriendo pero volví. Y volvi a esperar a mi tía y no es la hermana de mi mamá pero yo le decía tía. Y a mi abuela imagínate que paso todo en ese momento que quedamos tomando mate igual.

-Como si nada...

-Como si nada, y yo no me animaba a decirle a nadie.

- ¿Le pudiste contar a tu mamá?

-Sí, después cuando me fui agarre la bici y llegue lo primero que hice fue agarrar el teléfono y le dije a mamá, y mamá empezó a llorar, no podía creer y me habló la amiga de ella, en el trabajo estaba mamá y le conté todo y después vino toda la familia a preguntarme cómo pasó y le conté todo como pasó y vos sabes que no hicieron denuncia que no hicieron nada, ¿sabes por qué? una porque no querian ensuciar como quien dice el apellido porque mi primo es doctor, y otro era abogado, no sé qué, pero ta, ¿por qué no hicieron la denuncia? Bueno quedo todo por eso. Por tres años no fuimos a la casa de mi abuela.

Luego volvieron a esa casa cuando sus padres se separaron, ella y su madre pasaron poco tiempo ahí hasta encontrar otro lugar donde vivir. Cuenta con mucha indignación que su abuelo en ese momento le 
dijo "qué cagada nos mandamos", frente a lo cual ella pensó "pero yo no había hecho nada". Tiene mucho resentimiento hacia este abuelo y sobre todo hacia la actitud que tomó la familia; no hicieron nada.

En relación a esta temática, investigaciones a nivel nacional e internacional (Abal, Cheroni, \& Leopold, 2005; Bodelón, 2003; Chesney-Lind, 2006) han señalado que las adolescentes mujeres comportan singularidades vinculadas al disciplinamiento de su cuerpo y fundamentalmente al terreno de la sexualidad. Estas investigaciones han concluído que la mayoría de estas adolescentes han sufrido de maltrato y abuso sexual en sus historias personales, aspectos que a veces se reeditan en las prácticas punitivas que sufren.

Nunca había estado en un lugar así, ni había tenido ningún vínculo con la Justicia Penal de Adolescentes. La asistente social le dijo que tenía que esperar un mes para hablar con el juez en la audiencia. Esto la desespera por estar tanto tiempo sin ver a su hijo y sin tener noticias de él. Piensa pedir permiso para llamar a su mejor amiga, pero aún no ha podido. En esta primera semana no ha hablado con nadie de su entorno cercano. No puedo dejar de pensar en lo angustiante del aislamiento inicial que está viviendo Lucrecia, en su primera semana. En ese contexto de aislamiento y encierro, me convierto en una suerte de contacto con el exterior, ese exterior prohibido y del que nada sabe.

Ante la angustia recurrió a la psiquiatra, quien le dio tranquilizantes que ella ya había consumido previo a tener a su hijo. Su vida cotidiana transcurre dentro de las rutinas de la institución. Se levanta y desayuna en el cuarto, luego son llamadas por orden a bañarse y a ordenar el cuarto que comparten con las demás compañeras y luego del mediodía, desde las 13:30 a las 19:00, tienen talleres y convivencia. Cada día igual al otro. Los talleres a los que ha asistido son peluquería y repostería, la propuesta educativa tiene un marcado sesgo de género.

-Pero también pasarás tiempo afuera o ¿estas mucho tiempo adentro?

-Sí, todos los días igual. De mañana te levantás a las 9 por ahí tenés la leche.

- En la celda o afuera?

-En la celda. Después cada una sale y se limpia ahí, se baña y entra de nuevo después ta te dan la comida $y$ a la una y media salimos todas para afuera.

-¿Al patio?

-Sí, ahí nomas viste y a las siete para dentro.
Ante la pregunta por el futuro inmediato surge el tema de la educación secundaria. Quiere salir, terminar el liceo y hacer algo, a su madre no la va a tener toda la vida, dice "Me gustaría trabajar como asistente social, porque ayudas".

\section{Lucrecia: comenzando su tercera semana}

Es otra, hace dos semanas que está en el Hogar. La angustia y la desesperación se han apoderado de su gran cuerpo. Se achica, mira hacia abajo y llora. En muchas ocasiones me cuesta oírla y este estado permanece durante toda la entrevista.

Me cuenta que en esta semana la cambiaron de cuarto y tuvo problemas con sus compañeras. Se enteraron de que el problema había sido con su madre y la cosa se complicó. Las madres son figuras muy valoradas, entre los códigos institucionales parece figurar no "dañarás a tu madre". Por lo que he visto y preguntado, en las visitas siempre están las madres y para cuidar a los hijos que quedan afuera, también son las madres las que se hacen cargo. Ellas al igual que nosotros (técnicos, jueces, sociedad) establecen categorías en función de los delitos que hayas cometido: dañar a tu propia madre ocupa el peor lugar. La peor sanción: el rechazo y la agresión de sus compañeras.

En esta semana, pudo hablar con su amiga que le dio noticias de su hijo; está bien, viviendo en la casa de su padre. Su madre no ha vuelto a la casa. En dos semanas el niño cumple un año, piensa que quizás lo puedan traer a visitarla. Empiezo a consolarla, no lo puedo evitar, ya no pienso ni en la investigación ni en nada. La veo cada vez más angustiada y me acuerdo de las palabras de la dirección, al referirse al lugar de los investigadores; "si vienen de afuera a descompensarlas, no lo podemos permitir". No quiero perjudicarla aún más de lo que ya está.

Dice no disfrutar de ninguna actividad, está muy triste. Por primera vez, fue al taller de costura, se hizo una vincha y le gustaría volver para hacerle un regalo a su hijo. También hizo gimnasia. Me cuenta al pasar que el otro día intento cortarse el brazo, necesitaba descargar esa rabia. Contra su cuerpo era la forma con menos consecuencias. En ese momento pienso en los cortes carcelarios, todas están cortadas ¿para no lastimar a otros se lastiman ellas mismas? Qué puedo hacer como investigadora frente a este relato, la impotencia se apodera de mí, me distraigo, y cambio de tema.

Tiene cara de dormida y le pregunto si estaba durmiendo la siesta. Me dice que sí, que está tomando mucha medicación y está todo el día con sueño, el otro día se le durmió la cara. La medicación psiquiátrica 
entra en escena, ayuda a conciliar el sueño y a qué otras cosas me pregunto. A portarse bien y no ser agresiva seguro, también está la variable del consumo de drogas la cual justifica casi que cualquier intervención psiquiátrica en la institución.

Al final de la entrevista, se encuentra calmada, podemos empezar a hablar de cosas más alentadoras y me cuenta que quiere terminar hasta cuarto año de liceo, por lo menos, sexto año sería lo mejor. Me doy cuenta que hablar conmigo la tranquiliza, una suerte de distracción que rompe la rutina, algo de esto me consuela a mí en mi desasosiego. Es terrible pensar lo poco que puedo hacer por ella.

\section{Lucrecia: en su cuarta semana}

Me asusta el deterioro y la tristeza con la que llega. Nuevamente quiere que la cambien de cuarto, dice que hay dos compañeras que le quieren pegar. No sólo en la habitación o en los espacios comunes, sino que también en el baño se ha tomado a golpes.

Todos estos conflictos con sus compañeras, sumado a que extraña a su hijo y a su madre, la sumergen en una angustia impresionante. Toda la entrevista llora. Me resulta muy difícil, me angustia mucho su relato y en todo momento siento que estoy intentando calmarla. Mi objetivos de investigación pasan a ser objetivos de contención emocional, quiere quedarse hablando y siento que la tengo que escuchar e intentar calmar.

-Una gurisa me tiene rabia porque piensa que yo le pegaba a mamá, dos me quieren pegar. En el cuarto, esas dos que me quieren pegar les hacen la cabeza a la que está en mi cuarto, ¿viste?, yo le pedí a la directora que me cambie de cuarto y por ahi me cambian.

-Ta bravo Lucrecia y no podes estar...

- Me siento mal, y mas mira, más que extraño a mi hijo que ahora el 20 cumple el año, mas que extraño a mi madre y todo esto...

Piensa en su hijo, la semana que viene cumplirá un año, no sabe si lo podrá ver. Se acuerda del día que nació y me cuenta de su primer año de vida, cuándo se sentó por primera vez, cómo se rió. Me resulta muy fácil empatizar con ella en esta temática, acabo de pasar por lo mismo, tengo un hijo pequeño. Dice que el niño dormía con ella y tomaba teta. Vuelve a aparecer el mismo miedo, que el niño no la conozca cuando ella salga.

El único contacto con el afuera en estas tres semanas han sido las charlas telefónicas con su amiga y las entrevistas conmigo. Sabe por ella que su madre e hijo están bien, la abuela visita al niño y le lleva cosas a la casa del padre. Pienso en el aislamiento que producen estas condiciones de internación, nadie la ha visitado y el único contacto con el afuera conocido son las conversaciones telefónicas con su amiga.

La entrevista se torna cada vez más difícil, me dice que pidió una gillette para matarse, "me quiero cortar, me quiero cortar las venas no aguanto más". A lo cual yo le pregunto si habló con alguien de lo que me está diciendo. Llora y no puede ni hablar, la trato de calmar.

Me insiste en que la cambien de cuarto, luego comienza a decirme una y otra vez que quiere que la internen en una clínica, quiere salir de ahí y estar sola. Quién soy yo y en qué la puedo ayudar comienza a ser tema, y por qué utilizo un grabador. La desconfianza se instala en el vínculo entre nosotras, es la primera vez que la siento amenazante, y no puedo olvidar que ella está amenazada por la situación que está viviendo.

-Pero viste, ¿la grabación para qué?

-Te acordás que yo te dije que iba a hacer un informe para la institución, una investigación acerca de cómo están uds acá.

- ¿Esto va para el juez?

-No, para el juez no, no ahora, lo que sí voy a hacer...

- ¿El INAU qué hace?

-El INAU es el que esta decidiendo sobre ustedes acá, lo que si voy a hacer Lucrecia, si vos me permitís, capaz que hablar con la directora y decirle que te vi muy triste, que si te pueden ayudar

-Que me cambien de cuarto...

Me doy cuenta que me está pidiendo ayuda en lo concreto de su vida cotidiana en el encierro y me pregunto qué puedo hacer por ella, me da vergüenza contarle el cuento del proyecto de investigación. Se me ocurre hablar con la directora una vez que hayamos terminado la entrevista.

Finalmente, hablamos de los aspectos legales. Tiene muchas dudas, ¿cuándo será la audiencia? ¿Cómo será? ¿Quién es el Juez/a? Le explico lo que puedo respecto a cómo funcionan los mecanismos del sistema penal juvenil, pero ignoro quién es el juez. Siento que en un momento se enoja conmigo, ella siente que debo ayudarla. ¿Qué hago ahí por ella? 
Me hace pensar en mi lugar ahí, tengo bastantes limitaciones; le digo que hablaré con la directora. Ese es mi compromiso.

\section{El después}

Salgo muy afectada de la entrevista. De hecho, ese día no me quedo mucho rato más. Pido para hablar con la directora y me recibe. Comenzamos a hablar de Lucrecia y de la mejor forma de ayudarla, mi intención es pensar junto con la directora esta situación particular. El tema de los golpes a su madre se filtró, todas lo saben y en su mayoría la rechazan. Se compromete a cambiarla nuevamente de cuarto, pero surge el tema de que sólo tienen un módulo por lo cual es muy difícil separarla en el resto de los espacios comunes.

En las sucesivas veces me entero de cosas de Lucrecia a través de las educadoras. Se lastimó mucho con una gillete y terminó internada en una clínica psiquiátrica, como ella quería. Luego de la audiencia y de obtener el alta psiquiátrica, la trasladaron a su ciudad, a una institución de amparo. No transcurrieron más de 45 días en esta institución, que seguramente fueron una eternidad en su vida.

\section{Reflexiones finales. Acerca de la investigación cualitativa en contextos de encierro con adolescentes mujeres en el SPJU}

\section{De escritura y reflexividad}

Las epistemologías feministas (Haraway, 1995) han advertido acerca de que los modos de escritura hegemónicos en la ciencia, reflejan formas masculinas de producir conocimiento basados en la objetividad y neutralidad del investigador. Lo cual se performa en modos abstractos e impersonales de narrar.

Esta es una de las tensiones que recorre este proyecto, cómo narrar y/o escribir acerca de las adolescentes mujeres privadas de libertad. La dimensión ética entra en juego, establecimos un pacto de confidencialidad con ellas, las adolescentes que están cumpliendo una medida judicial en el encierro. El motivo: no vulnerabilizar aún más sus vidas con elementos que pueden ser institucionalmente mal usados. Una responsabilidad que pesa y mucho a la hora de escribir.

A lo que se suma, el lugar de la investigadora en la escritura del informe y/o tesis, muchas veces pretendidamente neutro y distante, cuando no ausente. Weisenfeld (2000) critica la ausencia del investigador en los reportes de investigación en cuestiones que hacen a su responsabilidad ética "a la responsabilidad que tiene al representar mediante su reportes a 'otros', de asignar significados a sus acciones, todo lo cual equivale a la mentalidad de la relación colonizadorcolonizado (Ulichny, 1997; Walsh-Bowers, 1995) o de iluminación" (Weisenfeld, 2000, p. 4).

Apostamos a un tipo de investigación cualitativa que muestra algo de las vicisitudes que conlleva la relación investigadora-investigado, donde los sentimientos vividos por la investigadora no son datos que contaminan sino que producen el conocimiento y donde la singularidad de un caso permite acercarse a la vida en un determinado contexto institucional.

Ahora bien, el contexto de encierro en el que se produce esta investigación pone en cuestión muchos de los postulados de la investigación cualitativa contemporánea, que hacen referencia a la horizontalidad, la simetría y la participación de las personas investigadas en el proceso de investigación (Sisto, 2008). La relación con el Otro en estos contextos, también sufre las marcas del encierro. Las entrevistas se producen en una habitación cerrada por fuera, la vivencia del encierro recorre las mismas y signa la relación investigadora-investigado. Si bien la participación es voluntaria y se explicita el para qué y el por qué de la misma, en muchos momentos surge por parte de las adolescentes el cuestionamiento acerca de quién es esa persona y qué puede hacer por mí en el aquí y ahora. Cuestionamiento que atraviesa a la investigadora y que la hace sentir frustración por lo poco que puede hacer en ese contexto, la apuesta es a futuro. Asesorar a la institución y formar operadores para el trabajo en esos contextos, objetivos proyectados de la investigación, pierden sentido cuando tenemos delante a un Otro sufriente.

Si bien, el contexto institucional vuelve difícil los planteamientos de simetría entre investigadoraadolescentes, sí creemos que debe habilitar procesos de diálogo y respeto en la relación que se establece con ellas. Con respecto a la escritura, tenemos la gran responsabilidad de hablar con ellas, los niveles de coautoría son muy difíciles de pensar en estos contexto, sin ausentarnos nosotras de lo que escribimos, como un ojo externo, poderoso y neutral.

\section{Lucrecia: acerca del género y el SPJU}

Con este proyecto de investigación, nos aventuramos al mundo de la investigación cualitativa en instituciones de encierro destinadas a adolescentes mujeres. Los motivos que fundamentan querer pensar el encierro en clave mujer tienen que ver, en primer término, con lo inexplorado de la temática sobre la 
que prima un modelo hegemónico masculino en las construcciones acerca de la infracción adolescente. En segundo término, partimos de la hipótesis de que el derecho penal tiene género, en este sentido la pregunta versa sobre cómo los sistemas penales funcionan para crear y recrear el género (Bodelón, 2003; Lauretis, 1989; Smart, 1992).

En este marco, por una parte consideramos al Derecho Penal como una tecnología privilegiada en la construcción del género, entendiendo al Derecho Penal como un universo simbólico, es decir un mundo de sentidos y significados cuyo alcance normativo depende de cómo se concreta en las diversas prácticas sociales. Por otra parte y siguiendo a Butler (2006), el género lo entendemos:

como una forma de hacer, una actividad incesante performada, en parte, sin saberlo y sin la propia voluntad, no implica que sea una actividad automática o mecánica. Por el contrario, es una práctica de improvisación en un escenario constrictivo. Además, el género propio no se "hace" en soledad. Siempre se está "haciendo" con otro o para otro, aunque el otro sea sólo imaginario. (p. 13)

El escribir acerca de Lucrecia como lo hemos hecho, nos permitió narrar el género desde una posición encarnada y posicionada en una determinada relación investigadora-adolescente, en un contexto institucional concreto. Las líneas que siguen a continuación, no intentan otra cosa que continuar narrando el género pero incluyendo las voces de otros investigadores que nos permiten contextualizar, ampliar y legitimar nuestra propia voz.

A través de Lucrecia, vemos como el género entra en escena desde la propia configuración institucional, existe un único lugar para mujeres adolescentes en todo el país. Esto se justifica en relación al escaso número de adolescentes mujeres que cumplen una medida de encierro en el SPJU, lo cual es un dato beneficioso pero que se constituye en una gran desventaja para las que sí cumplen una sanción con encierro, dado que deben ser sometidas a traslados a grandes distancias de sus lugares de residencia y no hay variabilidad en las características del centro como pasa con los varones, de más abiertos a más cerrados (Aloisio, Chouhy, Trajtenberg, \& Vigna, 2009).

¿Qué pasa con Lucrecia en sus primeras semanas de institucionalización? No sabe nada del mundo exterior, de su familia, de su hijo, la desesperación se apodera de ella. Luego de un tiempo, le habilitan las llamadas telefónicas: restrictivas y controladas. En un país pequeño, es trasladada para cumplir su pena, el aislamiento se potencia con la distancia geográfica de su lugar de residencia, lo que torna muy difícil las visitas del exterior.

Existe la hipótesis de que los sistemas penales se comportan de forma más benevolente con las mujeres, lo que explicaría el número menor de mujeres encarceladas, en el caso de las adolescentes las diferencias son abrumadoras, son aproximadamente el $5 \%$ de la población de adolescentes que cumplen una medida judicial en el encierro, según datos institucionales. De todas formas, diversas investigaciones sobre la temática (Carlen \& Worrall, 2004; Larrauri, 1994) sostienen que esta hipótesis no se aplica a las mujeres que no responden a los estándares de femineidad exigidos desde lo social y que en el caso de las mujeres existen controles sociales informales más eficaces que el control penal.

Esta doble desviación a la normatividad penal y de género, lleva a que nos preguntemos: ¿qué pasa con Lucrecia que está cumpliendo una pena por un delito de violencia familiar contra su madre? A la sanción del derecho penal, se le suma el castigo de sus propias compañeras y la pena que cae sobre su propia maternidad, es separada de su hijo. Sin duda que ha roto con lo esperado en los roles de hija y madre, en relación a las exigencias de género pautadas desde lo social. Se convierte en una hija que golpea a su madre y en una madre que abandona a su hijo. Si bien, la maternidad adolescente es un tema recurrente para los sistemas penales juveniles, no está previsto en el dispositivo institucional pautas claras para que las adolescentes puedan tener a sus hijos con ellas.

La tensión singularizar-generalizar nos ha atravesado en toda la narración. No pretendemos saldar la discusión de la generalización a través de un caso, dado que para construir nuestro trabajo de campo hemos utilizado nueve, pero pretendemos mostrar como a través de una singularidad se pueden pensar algunas particularidades del encierro en adolescentes mujeres.

Con Lucrecia hemos podido comprender algunas de las vicisitudes de la vida en el encierro para una adolescente mujer en el SPJU. Asimismo, nos hemos enfrentado a las dificultades de realizar una investigación cualitativa en el marco de una institución de reclusión. Lucrecia nos habilita a reflexionar acerca de otras prácticas de escritura, que al decir de Billig (1994) vuelven a las reconstrucciones particulares que lejos de reducciones individualistas permiten otros tránsitos a las generalizaciones. Con esto buscamos nuevas retóricas que sirvan para poblar nuestras narrativas de investigación, donde la reflexividad es una condición fundamental en la producción de conocimiento. 


\section{Nota}

1 Institución rectora en política de infancia y adolescencia.

\section{Referencias}

Abal, A., Cheroni, A., \& Leopold, S. (2005). Adolescencia e infracción: una aproximación a la construcción subjetiva. Montevideo: INAU-Cenfores.

Aloisio, C., Chouhy, C., Trajtenberg, N., \& Vigna, A. (2009). Jóvenes en conflicto con la ley: una mirada a las instituciones de rehabilitación desde la perspectiva de género. Montevideo: MIDES - INFAMILIA.

Billig, M. (1994). Repopulating the depopulated pages of Social Psychology. Theory and Psychology, 4(3), 307-335.

Bodelón, E. (2003). Género y sistema penal: los derechos de las mujeres en el sistema penal. In R. Bergalli (Ed.), Sistemas penales y problemas sociales (pp. 451-486). Valencia: Tirant lo Blanch.

Butler, J. (2006). Deshacer el género. Barcelona: Paidós.

Carlen, P. \& Worrall, A. (2004). Analysing women's imprisonment. London: Willan Publishing.

Chesney-Lind, M. (2006). Patriarchy, crime and justice. Feminist Criminology in an era of backlash. Feminist Criminology, 1(1), 6-26.

Denzin, N. \& Lincoln, I. (2003). The landscape of qualitative research. Theories and Issues. Thousand Oaks, CA: Sage.

Devereux, G. (1989). De la ansiedad al método en las ciencias del comportamiento. México: Siglo XXI Editores. (Original publicado en 1977)

Donzelot, J. (2008). La policía de las familias. Buenos Aires: Nueva Visión. (Original publicado en 1977)

García, F. V. (2006). El problema de la reflexividad en Pierre Bourdieu de la Epistemología a la Ética. Opinión Jurídica, 5(10), 87-104

Hammersly, M. \& Atkinson, P. (1994). Etnografia. Métodos de investigación. Barcelona: Paidós. (Original Publicado en 1983)

Haraway, D. (1995). Ciencia, cyborgs y mujeres. La reinvención de la naturaleza. Madrid: Cátedra.

Igareda, N. (2009). La maternidad de las mujeres presas. In E. Bodelón \& G. Nicolás (Comps.), Género y dominación. Críticas feministas del derecho y el poder (pp. 159 -194). Barcelona: Anthropos.

Iñiguez, L. (2003). Análisis del discurso. Manual para las ciencias sociales. Barcelona: UOC.

Larrauri, E. (1994). Mujeres, derecho penal y criminología. Madrid: Siglo Veintiuno Editores.

Lauretis, T. (1989). Technologies of gender. Essays on theory, film and fiction. Londres: Macmillan Press.

Ley $n^{o .}$ 17.823, de 7 de setiembre de 2004. (2004). Código de la Niñez y Adolescencia. Acceso en 13 de junio, 2011, en http://www0.parlamento.gub.uy/leyes/AccesoTextoLey. asp?Ley $=17823 \&$ Anchor $=$

Olabuénaga, J. L. R. (2009). Metodología de la investigación cualitativa. Bilbao: Universidad de Deusto. (Original publicado en 1996)

Patai, D. (1991). U.S. Academics and Third World Women: Is ethical research possible? In S. B. Gluck \& D. Patai (Eds.), Women's words: The feminist practice of oral history (pp. 137-153). New York: Routledge.
Pillow, W. (2003). Confession, catharsis, or cure? Rethinking the uses of reflexivity as methodological power in qualitative research. Qualitative Studies in Education, 16(2), 175-196.

Sisto, V. (2008). La investigación como una aventura de producción dialógica: la relación con el otro y los criterios de validación en la metodología cualitativa contemporánea. Psicoperspectivas, 7, 114-136.

Smart, C. (1992). La teoría feminista y el discurso jurídico. In H. Birgin (Ed.), El Derecho en el Género y el Género en el Derecho (pp. 31- 71). Buenos Aires: Biblos.

Spivak, G. (1988). Can the subaltern speak? In C. Nelson \& L. Grossberg (Eds.), Marxism and the interpretation of culture (pp. 271- 313). Urbana, IL: University of Illinois Press.

Taylor, S. J. \& Bogdan, R. (1992). Introducción a los métodos cualitativos de investigación. La búsqueda de significados. Barcelona: Paidós. (Original publicado en 1984)

Walsh-Bowers, R. (1995). The reporting and ethics of the research relationship in areas of interpersonal psychology, 1939-89. Theory and Psychology, 5, 233-250.

Weisenfeld, E. (2000). Entre la prescripción y la acción: La brecha entre la teoría y la práctica en las investigaciones cualitativas. Forum: Qualitative Social Research, 1(2), Art. 30. Acceso en 19 de maio, 2012, en http://www.qualitativeresearch.net/index.php/fqs/article/view/1099/2419

Submissão em: 19/06/2013

Revisão em: 12/07/2014

Aceite em: 01/09/2014

Laura López Gallego es licenciada en Psicología por la Facultad de Psicología de la Universidad de la República (UdelaR). Magister en Psicología Social por el Departamento de Psicología Social de la Universidad Autónoma de Barcelona (UAB). Doctoranda en Psicología Social del Departamento de Psicología Social de la UAB. Docente e investigadora del Instituto de Psicología Social de la Facultad de Psicología de la UdelaR. Investigadora activa del Sistema Nacional de Investigadores de la Agencia Nacional de Investigación e Innovación. Dirección: Tristán Narvaja 1674, C.P. 11200. Montevideo, Uruguay. E-mail: 1lopez@psico.edu.uy

\section{Como citar:}

López Gallego, L. (2014). Proceso de reflexividad en un contexto de privación de libertad de adolescentes mujeres. Psicologia \& Sociedade, 26(3), 603-612. 